BMJ Open Ophthalmology

\title{
Comparing vision and macular thickness in neovascular age-related macular degeneration, diabetic macular oedema and retinal vein occlusion patients treated with intravitreal antivascular endothelial growth factor injections in clinical practice
}

\author{
Rajya L Gurung (D) , ' Liesel M FitzGerald, ${ }^{1}$ Bennet J McComish (D) ,' \\ Alex W Hewitt, ${ }^{1}$ Nitin Verma, ${ }^{2}$ Kathryn P Burdon ${ }^{1}$
}

To cite: Gurung RL, FitzGerald LM, McComish BJ, et al. Comparing vision and macular thickness in neovascular age-related macular degeneration, diabetic macular oedema and retinal vein occlusion patients treated with intravitreal antivascular endothelial growth factor injections in clinical practice. BMJ Open Ophthalmology 2021;6:e000749. doi:10.1136/ bmjophth-2021-000749

- Additional online supplemental material is published online only. To view, please visit the journal online (http://dx.doi.org/10.1136/ bmjophth-2021-000749).

Received 22 February 2021 Revised 17 March 2021 Accepted 24 April 2021

\section{Check for updates}

(c) Author(s) (or their employer(s)) 2021. Re-use permitted under CC BY-NC. No commercial re-use. See rights and permissions. Published by BMJ.

For numbered affiliations see end of article.

Correspondence to Dr Rajya L Gurung; rajyalaxmi. gurung@utas.edu.au

\section{ABSTRACT}

Objective To compare the visual outcomes of intravitreal antivascular endothelial growth factor (antiVEGF) injections in neovascular age-related macular degeneration (nAMD), diabetic macular oedema (DMO) and retinal vein occlusion (RVO) in a real-world setting.

Methods and analysis Retrospective analysis of data from the Tasmanian Ophthalmic Biobank database. The median change in best-corrected visual acuity (BCVA) between baseline and 12 months post initiating intravitreal anti-VEGF treatment were compared between the three diseases. Final BCVA, central macular thickness (CMT), cumulative number of injections and overall predictors of change in BCVA and CMT were also determined.

Results At 12 months, change in BCVA was significantly different between nAMD, DM0 and RV0 cohorts $(p=0.032)$, with lower median change for DM0 (2 letters, range -5 to 20) than for RV0 (11 letters, range -20 to 35). Likewise, CMT change was significantly different between the three cohorts $(p=0.022)$, with a smaller reduction in CMT in DMO $(-54 \mu \mathrm{m}$, range -482 to 50$)$ than RV0 patients $(-137 \mu \mathrm{m}$, range -478 to $43 ; p=0.033)$. Total number of injections received $(p=0.028)$ and final BCVA score $(p=0.024)$ were also significantly different between the groups. Baseline BCVA was a negative predictor $(p=0.042)$ and baseline CMT a positive predictor $(p<0.001)$ of outcome. After adjusting for baseline BCVA and CMT, diagnosis of nAMD or RVO was a predictor of visual improvement compared with the DMO.

Conclusions At the end of 12 months, nAMD and RVO cohorts had the greatest improvement in BCVA, however the final BCVA for DMO was significantly better than for nAMD.

\section{INTRODUCTION}

Antivascular endothelial growth factor (anti-VEGF) intravitreal injections have revolutionised the treatment of retinal

\section{Key messages}

What is already known about this subject?

> Vascular endothelial growth factor (VEGF) is the common biological determinant for neovascular age-related macular degeneration (nAMD), diabetic macular oedema (DMO) and retinal vein occlusion (RVO).

Intravitreal anti-VEGF injections are the standard of care for nAMD, DMO and RVO.

What are the new findings?

DM0 patients had lower improvement in vision compared with RVO and nAMD.

Final vision for DMO and RVO was similar, and better than for nAMD.

How might these results change the focus of research or clinical practice?

- Anti-VEGF injections are beneficial for most DMO patients in stabilising their vision despite having a lower improvement.

Diagnosing and treating $\mathrm{nAMD}$ and RVO patients at an earlier time point might improve their final vision further.

conditions. Since the advent of the first intravitreal anti-VEGF injection in $2000,{ }^{1}$ it has become frontline treatment for many ocular conditions $^{2}$ including neovascular age-related macular degeneration (nAMD), diabetic macular oedema (DMO) and macular oedema in retinal vein occlusion (RVO), all major causes of central visual impairment.

AMD is the leading cause of irreversible blindness in older populations. ${ }^{3}$ In nAMD, neovascularisation underlying the choroid breaks through Bruch's membrane into the 
retinal pigment layer, leading to the formation of the choroidal neovascular membrane. Though the exact trigger initiating the cascade of new vessel formation is unknown, hypoxia, inflammation and complement activation are believed to play pivotal roles in the pathogenesis of nAMD. ${ }^{4}$ There is also an increased production of VEGF, which leads to angiogenesis and increased vascular permeability. Studies of eye autopsies from nAMD patients show increased VEGF levels in the retinal pigment epithelium and choroidal blood vessels of the macula. ${ }^{5} \mathrm{DMO}$, a retinal complication of diabetes mellitus, is the leading cause of vision impairment in the working-age population. ${ }^{6}$ The breakdown of the bloodretinal barrier, with leakage of fluid from the retinal micro-vasculature, is a major pathogenic mechanism for DMO, mediated principally by VEGF. ${ }^{7}$ VEGF protein levels are significantly elevated in both the aqueous and vitreous humour of DMO eyes ${ }^{89}$ and increased immunostaining of VEGF has been demonstrated in diabetic retinas. ${ }^{10} \mathrm{RVO}$ is the second most common type of retinal vascular disorder after diabetic complications. ${ }^{11}$ Vascular occlusion in the acute phase of RVO leads to retinal hypoxia, which causes an increase in VEGF production and results in disruption of the blood-retinal barrier, increased vascular permeability and macular oedema. ${ }^{12}$

Although nAMD, DMO and RVO have independent and complex etiopathogeneses, an increase in VEGF is observed across all three conditions. ${ }^{5812}$ Consequently, anti-VEGF agents are the first line of treatment. Very few studies have compared real world treatment outcomes of this therapy in the three patient groups. This study aimed to compare the clinical outcomes of anti-VEGF treated nAMD, DMO and RVO patients in a real-world, clinicbased setting.

\section{MATERIALS AND METHODS}

\section{Study design}

This was a retrospective multicentre analysis of patients from the Tasmanian Ophthalmic Biobank. The Biobank is an initiative of the Menzies Institute for Medical Research, University of Tasmania, and associated clinics that aims to collect DNA samples and clinical information from Tasmanian residents diagnosed with a variety of ocular conditions. Written informed consent was obtained from each participant prior to enrolment in the biobank. Patients were not involved in the design or conduct of this study.

\section{Participants}

Biobank participants with a diagnosis of nAMD, DMO or RVO and receiving anti-VEGF therapy between 2013 and 2019 in public or private eye clinics in Tasmania were included. nAMD patients were known choroidal neovascular cases, diagnosed secondary to AMD as demonstrated by fluorescein angiography and central macular thickness $(\mathrm{CMT}) \geq 315 \mu \mathrm{m}$ measured by spectral domain optical coherence tomography (SD-OCT; Heidelberg Spectralis; Heidelberg Engineering, Heidelberg, Germany).
DMO patients were those with clinically diagnosed centre-involving DMO and CMT $\geq 315 \mu \mathrm{m}$ as measured by SD-OCT. RVO patients were those who presented with macular oedema secondary to clinically diagnosed RVO and CMT $\geq 315 \mu \mathrm{m}$. Eyes with cysts in the central 1000 $\mu \mathrm{m}$, fresh haemorrhages, definitive leakage suggestive of a neovascular membrane in fluorescein angiography but with CMT $<315 \mu \mathrm{m}$, were also included in this study if they met the diagnostic criteria for one of the three diseases. To be included, patients must have received a loading dose of at least three consecutive anti-VEGF injections at intervals of 4-6 weeks and a follow-up of 12 months from initiation of anti-VEGF injections. Patients who received any systemic anti-VEGF therapy, intra-ocular steroid or vitreoretinal surgery in the 6 months before the first injection, had severe media opacity obscuring detailed fundus evaluation, and/or had follow-up data for less than 12 months were excluded from the study. DMO patients who had received laser eye therapy before or during anti-VEGF injections were still included in the study as we aimed to evaluate outcomes driven by clinical decision making rather than strict trial protocols. Treatment decisions, including choice of anti-VEGF drug and switching between agents, were at the discretion of the treating physician. Where bilateral anti-VEGF injections were given, data from the better responding eye were used. Patients were also required to have complete data for demographic and clinical characteristics (baseline visual acuity, baseline CMT, number of injections, laterality of eye, lens status, age, sex, smoking history, hypertensive status, lipid profile and disease type).

\section{Clinical data collection}

Clinical data were collected retrospectively from a review of medical records for every injection for 12 months after the date of the first injection. The data included ophthalmologic diagnoses, best-corrected visual acuity (BCVA), OCT measurements, intraocular pressure, lens status, laterality of affected eye, anti-VEGF injections (type and number), age, sex, hypertensive status, lipid profile, smoking history and adverse drug events post anti-VEGF injection. BCVA was recorded using Snellen's visual acuity score and was converted to early treatment diabetic retinopathy study (ETDRS) letter scores using the formula ETDRS $=85+\left[50 \times \log _{10}\right.$ (Snellen acuity fraction) $].{ }^{13}$ CMT was measured using SD-OCT. Change in BCVA and CMT were defined as the difference between BCVA or CMT immediately prior to the first injection and measurements taken at the 12-month follow-up visit. The injection number was the total number of injections received at the end of 12 months from the date of the first injection. Patients taking antihypertensive or lipid-lowering drugs were classified as hypertensive or hyperlipidaemic respectively.

\section{Outcome measures}

The primary outcome was change in BCVA at 12 months after the first intravitreal anti-VEGF injection. Secondary 
outcomes included change in CMT, final BCVA, final CMT and the cumulative number of injections over 12 months. These outcomes were compared between the three disease groups of nAMD, DMO and RVO.

To explore the data further, we categorised all participants into functional and anatomical responder or non-responder groups. A functional responder was defined as (1) an improvement of 5 ETDRS letters or more from the baseline or (2) 15 ETDRS letters or more improvement from baseline BCVA. An anatomical responder was defined as a $10 \%$ or greater reduction in CMT from the baseline. We also investigated possible clinical and demographic factors predicting the functional and anatomical responses. Outcome stratified by injection subtypes were also evaluated.

\section{Statistical analysis}

Statistical analyses were performed using SPSS version V.26 (SPSS IBM). Numerical variables are described using the mean with $\mathrm{SD}$, and median with range. The normality of all quantitative variables was assessed using the Kolmogorov-Smirnov test. Parametric tests were applied to normally distributed outcome variables and nonparametric tests to those that were not. Betweengroup analyses of the three disease types were done using the Kruskal-Wallis test for continuous variables and the $\chi^{2}$ test for categorical variables. Fisher's exact test was used for categorical variables when sample size was small. Univariable and multivariable binomial logistic regression models were used to explore the effects of covariates (age, baseline BCVA, baseline CMT, number of injections, disease type) on responder status at 12 months. Variables selected for the multivariable analyses were those that were statistically significant in univariable analyses, as well as those reported in previous studies. Firth's logistic regression was used when the dichotomised outcome was rare in any of the disease groups. Sex (male:female), current or past smoking status (yes:no), hypertension (yes:no), hyperlipidaemia (yes:no) and lens status (phakic:pseudophakic) were dichotomised for statistical analyses. Tests were considered significant at $\mathrm{p}<0.05$ after Bonferroni correction for multiple testing.

\section{RESULTS}

\section{Baseline and clinical characteristics}

A total of 243 patients receiving anti-VEGF injections were identified in the Tasmanian Ophthalmic Biobank. Of these, 50 nAMD, $37 \mathrm{DMO}$ and $30 \mathrm{RVO}$ patients met the inclusion criteria with sufficiently complete data. The overall baseline and clinical characteristics are summarised in table 1 . The median baseline BCVA was significantly higher for the DMO cohort (70 letters, range 0-80) compared with the nAMD (55 letters, range $0-80$ ) and RVO cohorts (55 letters, range $0-84$; $\mathrm{p}=0.002)$. Median baseline CMT was higher in the RVO (483.5 $\mu \mathrm{m}$, range 263-763) cohort compared with the two other cohorts $(\mathrm{p}=0.007)$. The proportion of pseudophakic participants was higher in the DMO (43\%) and
nAMD (54\%) cohorts compared with the RVO cohort, $(10 \% ; \mathrm{p}<0.001)$. The nAMD cohort was older than the other two groups, $(p<0.001)$. Comorbidities (hypertension, hyperlipidaemia) were present in a significantly higher proportion of DMO (92\% and $86 \%$, respectively) patients compared with the other cohorts $(p<0.001)$. Gender, laterality of eye and proportion of patients receiving different types of injections (Bevacizumab, Genentech; Ranibizumab, Novartis; Aflibercept, Regeneron) did not differ significantly between the three cohorts. The majority of patients received either bevacizumab or a combination of anti-VEGF drugs over the 12 months period (table 1). No serious ocular or systemic adverse events were noted post anti-VEGF injection in any of the disease groups.

\section{Outcome measures at the end of $\mathbf{1 2}$ months according to disease type}

After 12 months of anti-VEGF treatment, BCVA change was significantly different between the three diseases, ( $\mathrm{p}=0.032$; table 2). Pairwise comparison indicated that this result was driven by the smaller change in DMO (two letters, range -5 to 20 ) than RVO patients (11 letters, range -20 to $35 ; \mathrm{p}=0.027$; table 2 ).

At 12 months, CMT change was also significantly different between the three diseases, $(p=0.022$; table 2) with a greater median CMT reduction in the RVO cohort $(-137 \mu \mathrm{m}$, range -478 to 43$)$ than the DMO cohort $(-54$ $\mu \mathrm{m}$, range -482 to $50 ; \mathrm{p}=0.033$ ).

The total number of injections received between baseline and 12 months was also significantly different between the three groups, $(\mathrm{p}=0.028$; table 2$)$. Pairwise comparison indicated that the median number of injections was significantly lower for DMO $(\mathrm{n}=9$, range $3-13)$ than $n A M D$ patients $(\mathrm{n}=10$, range $5-17 ; \mathrm{p}=0.019)$.

There was also a significant difference in final BCVA between the three diseases $(p=0.024$; table 2$)$, with a higher median final BCVA in the DMO cohort (72 letters, range 0-85) compared with the nAMD group (66.5 letters, range $20-85 ; \mathrm{p}=0.019$ ). There was no significant difference in final CMT between the three diseases $(\mathrm{p}=0.242$; table 2$)$.

\section{Differences in outcome measures based on functional and anatomical response}

Stratifying outcome on the basis of functional response revealed significant differences in the proportion of patients from each cohort that improved by at least 5 ETDRS letters ( $\mathrm{p}=0.003$; table 3 ) and by 15 ETDRS letters $(p<0.001)$. In both instances, these results were driven by the DMO cohort $(32 \%$ and $5 \%$, respectively) where visual improvement was lower than that observed in the nAMD (62\% and $32 \%$, respectively) or RVO cohorts $(70 \%$ and $43 \% ; \mathrm{p}<0.05)$. Analyses based on anatomical response found no significant differences between the three disease cohorts, $(\mathrm{p}=0.122$; table 3). 
Table 1 Baseline and clinical characteristics of patients in each disease cohort

\begin{tabular}{|c|c|c|c|c|}
\hline Variables & nAMD $(\mathrm{N}=50)$ & $\mathrm{DMO}(\mathrm{N}=37)$ & $\operatorname{RVO}(\mathrm{N}=30)$ & P value* \\
\hline Baseline BCVA (ETDRS letters) & $55(0-80)$ & $70(0-80)$ & $55(0-84)$ & $0.002 \dagger$ \\
\hline Baseline CMT $(\mu \mathrm{m})$ & $353.5(199-794)$ & $352(276-987)$ & $483.5(263-763)$ & $0.007 \ddagger$ \\
\hline Lens status (\% Pseudophakic) & 54 & 43 & 10 & $<0.001 \S$ \\
\hline Age (years) & $80(61-98)$ & $71(52-88)$ & $75(45-90)$ & $<0.0019$ \\
\hline Male (\%) & 54 & 40 & 43 & 0.414 \\
\hline Laterality of eye (\% R) & 54 & 57 & 33 & 0.115 \\
\hline Hypertension (\% positive) & 64 & 92 & 73 & $0.011^{\star \star}$ \\
\hline Hyperlipidaemia (\% positive) & 52 & 86 & 43 & $<0.001 \dagger \dagger$ \\
\hline Smoker (\%) & 72 & 54 & 50 & 0.091 \\
\hline \multicolumn{5}{|l|}{ Injection type } \\
\hline Bevacizumab (\%) & 38 & 54 & 57 & $>0.05$ \\
\hline Ranibizumab (\%) & 8 & 3 & 13 & $>0.05$ \\
\hline Aflibercept (\%) & 10 & 8 & 0 & $>0.05$ \\
\hline Mixed (\%) & 44 & 35 & 30 & $>0.05$ \\
\hline Diabetes duration (years) & - & $23(2-50)$ & - & - \\
\hline $\mathrm{HbA1c}(\mathrm{mg} / \mathrm{dL})$ & - & $7.8(5.9-12.1)$ & - & - \\
\hline Laser at baseline (\% positive) & - & 57 & - & - \\
\hline
\end{tabular}

Data are presented as medians (range) for continuous data and proportions for categorical data.

${ }^{*} P$ values are global $p$ values testing for a difference between any of the diseases. (Kruskal-Wallis for continuous variables or $\chi^{2} /$ Fisher's exact for categorical). For comparisons that are significant at the global level, adjusted pairwise $p$ values for individual study comparisons are reported.

Significant $p$ values are bolded.

†nAMD vs DMO $p=0.002$; DMO vs RVO $p=0.020$; $n A M D$ vs RVO $p=1.000$

fnAMD vs DMO $p=1.000 ;$ DMO vs RVO $p=0.027$; $A M D$ vs RVO $p=0.008$

$\S n A M D$ vs DMO $p>0.05$; DMO vs RVO $p<0.05$; nAMD vs RVO $p<0.05$.

InAMD vs DMO $p<0.001$; DMO vs RVO $p=0.858$; nAMD vs RVO $p=0.005$.

**AMD vs DMO $p<0.05$; DMO vs RVO $p>0.05 ; n A M D$ vs RVO $p>0.05$.

††nAMD vs DMO $p<0.05$; DMO vs RVO $p<0.05$; nAMD vs RVO $p>0.05$

BCVA, best-corrected visual acuity; CMT, central macular thickness; DMO, diabetic macular oedema; ETDRS, early treatment diabetic retinopathy

study; Mixed, combination of either of the three injection types; nAMD, neovascular age-related macular degeneration; RVO, retinal vein occlusion.

Outcome stratified by injection type

When stratified by injection type, 'bevacizumab' or 'mixed injection' showed greater improvements in the RVO group and less improvement in the DMO group after 12 months, (online supplemental file S1). A separate analysis for 'ranibizumab' and 'aflibercept' was not done due to the size of the cohort (table 1).

\section{Predictors of functional response}

Table 4 summarises the results of logistic regression investigating the influence of independent variables on functional and anatomical response. Whether using the definition of at least 5 or 15 ETDRS letters improvement, functional response was associated with baseline BCVA and disease type. Patients with a higher baseline BCVA

Table 2 Outcome measures at the end of 12 months according to disease type

\begin{tabular}{|c|c|c|c|c|}
\hline Variables & nAMD (N=50) & DMO (N=37) & RVO $(\mathrm{N}=30)$ & P value* \\
\hline BCVA change (ETDRS letters) & 5 (-30 to 40$)$ & $2(-5$ to 20$)$ & $11(-20$ to 35$)$ & $0.032 \dagger$ \\
\hline CMT change $(\mu \mathrm{m})$ & $-41.5(-340$ to 81$)$ & $-54(-482$ to 50$)$ & $-137(-478$ to 43$)$ & $0.022 \ddagger$ \\
\hline No of Injections & $10(5$ to 17$)$ & 9 (3 to 13$)$ & $10(5$ to 13$)$ & $0.028 \S$ \\
\hline Final BCVA (ETDRS letters) & 66.5 (20 to 85$)$ & $72(0$ to 85$)$ & $68(0$ to 80$)$ & $0.024 \rrbracket$ \\
\hline Final CMT $(\mu \mathrm{m})$ & 282.5 (195 to 551$)$ & 296 (226 to 532) & 288 (222 to 710$)$ & 0.242 \\
\hline
\end{tabular}

Data are medians (range).

${ }^{*} \mathrm{P}$ values are global $\mathrm{p}$ values testing for a difference between any of the diseases. (Kruskal-Wallis for continuous variables). For comparisons that are significant at the global level, adjusted pairwise $p$ values for individual study comparisons are reported. Significant $p$ values are bolded.

tnAMD vs DMO $p=0.494$; DMO vs RVO $p=0.027$; $A M D$ vs RVO $p=0.417$

łnAMD vs DMO $p=1.000$; DMO vs RVO $p=0.033$; $n A M D$ vs RVO $p=0.046$.

§nAMD vs DMO $p=0.019 ;$ DMO vs RVO $p=0.498 ; n A M D$ vs RVO $p=1.000$.

InAMD vs DMO $p=0.019$; $D M O$ vs RVO $p=0.498 ; n A M D$ vs RVO $p=0.834$

BCVA, best-corrected visual acuity; CMT, central macular thickness; DMO, diabetic macular oedema; ETDRS, early treatment diabetic retinopathy study; nAMD, neovascular age-related macular degeneration; RVO, retinal vein occlusion. 


\begin{tabular}{llccc}
\hline \multicolumn{1}{l}{ Table 3} & \multicolumn{1}{l}{ Comparing functional and anatomical response in different diseases } & & \\
\hline Variables & nAMD (N=50) & DMO (N=37) & RVO (N=30) & P value* $^{*}$ \\
\hline$\geq 5$ ETDRS letters increase (\%) & $31(62)$ & $12(32)$ & $21(70)$ & $\mathbf{0 . 0 0 3 \dagger}$ \\
$\geq 15$ ETDRS letters increase (\%) & $16(32)$ & $2(5)$ & $13(43)$ & $<0.005 \ddagger$ \\
$\geq 10 \%$ decrease in CMT (\%) & $27(54)$ & $24(65)$ & $23(77)$ & 0.122 \\
\hline
\end{tabular}

Data are presented as the number of individuals and corresponding percentages.

${ }^{*}$ Global $p$ values testing for a difference between any of the diseases with a $\chi^{2}$ test are given. For comparisons that are significant at the global level, adjusted pairwise $p$ values for individual study comparisons are reported. Significant $p$ values are in bold.

†nAMD vs DMO p<0.05; DMO vs RVO p<0.05; nAMD vs RVO $p>0.05$.

łnAMD vs DMO p<0.05; DMO vs RVO p<0.05; nAMD vs RVO p >0.05.

CMT, central macular thickness; DMO, diabetic macular oedema; ETDRS, early treatment diabetic retinopathy study; nAMD, neovascular age-

related macular degeneration; RVO, retinal vein occlusion.

had a lower likelihood of 5 or 15 letters improvement under a univariable $(\mathrm{p}=0.001, \mathrm{p}<0.001$, respectively) and multivariable model $(\mathrm{p}=0.042, \mathrm{p}<0.001$, respectively). Conversely, nAMD and RVO patients had a higher likelihood of 5 or 15 ETDRS letters improvement when compared with DMO patients under a univariable model, a difference which remained significant for RVO patients under a multivariable model $(\mathrm{p}=0.038, \mathrm{p}=0.004$, respectively; table 4). Baseline CMT was also associated with improvements of 5 or 15 ETDRS letters under a univariable model $(\mathrm{p}=0.030$, $\mathrm{p}=0.041$, respectively); however, these associations were no longer significant when a multivariable model was applied.

\section{Predictors of anatomical response}

Baseline CMT was positively associated with anatomical response defined as at least a $10 \%$ reduction in CMT (table 4). Patients with higher baseline CMT had a greater likelihood of a $10 \%$ reduction in CMT under both a univariable $(\mathrm{p}<0.001)$ and multivariable model $(p<0.001)$. There was also evidence that a high baseline BCVA was associated with a poor CMT response $(\mathrm{p}=0.029)$; however, this result was not significant once other covariates were added to the model (table 4).

\section{DISCUSSION}

This study shows that nAMD, DMO and RVO patients treated with anti-VEGF therapy have significantly different treatment outcomes. Participants with DMO experienced the smallest gains in vision compared with nAMD or RVO patients. Low baseline BCVA was a positive predictor for improvement in vision while high baseline CMT predicts greater reduction in thickness. After adjusting for baseline BCVA and CMT, diagnosis of RVO was a predictor of better visual outcomes when compared with the diagnosis of DMO.

All three diseases showed less vision improvement than randomised controlled trials (RCTs) testing the effectiveness of anti-VEGF therapy. Trials for DMO reported 5.9-13.3 ETDRS letters gained, ${ }^{14}{ }^{15}$ compared with only two letters in the current study. Similarly, the improvements of 5 and 11 letters for nAMD and RVO, respectively, are lower than the respective 6.5-9 and 16.4-18.3 letters improvements in reported trials. ${ }^{1617}$ This phenomenon is not unique to our study with similar findings reported in other real-world and observational studies. ${ }^{18} 19$ This could be because clinical trials have strict eligibility criteria, treatment and follow-up schedules and exclude patients with extremely poor baseline characteristics or comorbidities. We report similar predictors of visual outcome as the clinical trials (baseline BCVA and CMT). ${ }^{2021}$

Our study shows similar levels of vision improvement in nAMD and RVO patients to other studies in realworld settings ${ }^{22}{ }^{23}$; however, our DMO patients did not show as much improvement as previous reports. ${ }^{24}$ The DMO patients in our study had better baseline vision than the other two groups. The association between baseline vision and VA change has been clearly demonstrated in prior research. ${ }^{25}$ Many studies have shown that good baseline vision is associated with smaller vision gains but better final vision. ${ }^{26}$ It is likely that the lack of BCVA improvement at 12 months in the DMO group is partly due to a ceiling effect, that is, they had less room for improvement. ${ }^{27}$ Anti-VEGF therapy in this group may primarily act to prevent progressive vision loss that would be expected without treatment. However, the results of the multivariable regression analysis indicated that the significant negative association of DMO with vision improvement persisted even after adjustment for baseline BCVA and baseline CMT. Therefore, the involvement of a ceiling effect in this study was relatively limited. Further, as noted by Dugel $e t a l$, a better starting vision does not always guarantee a ceiling effect. ${ }^{25}$ Many RCTs have also shown improvement in vision ( $\geq 5$ ETDRS letters) despite better baseline vision. ${ }^{25} 28$

Conversely, nAMD and RVO patients had significantly lower baseline BCVA than DMO patients but the final BCVA was similar to DMO in the RVO group and only slightly lower in the nAMD group. It has previously been reported that worse baseline vision is associated with better visual gains but worse final vision. ${ }^{29}$ However, this was not the case in the RVO and nAMD groups in our study, with both groups achieving satisfactory endpoints despite worse starting vision. The slightly poorer final vision in nAMD could be due to less underlying damage in the RVO group, given that $\mathrm{nAMD}$ is the late stage of a progressive disease. Further, the DMO group in our study was significantly younger than the other two 
Table 4 Univariable and multivariable logistic regression analyses investigating factors predictive of functional and anatomical responses

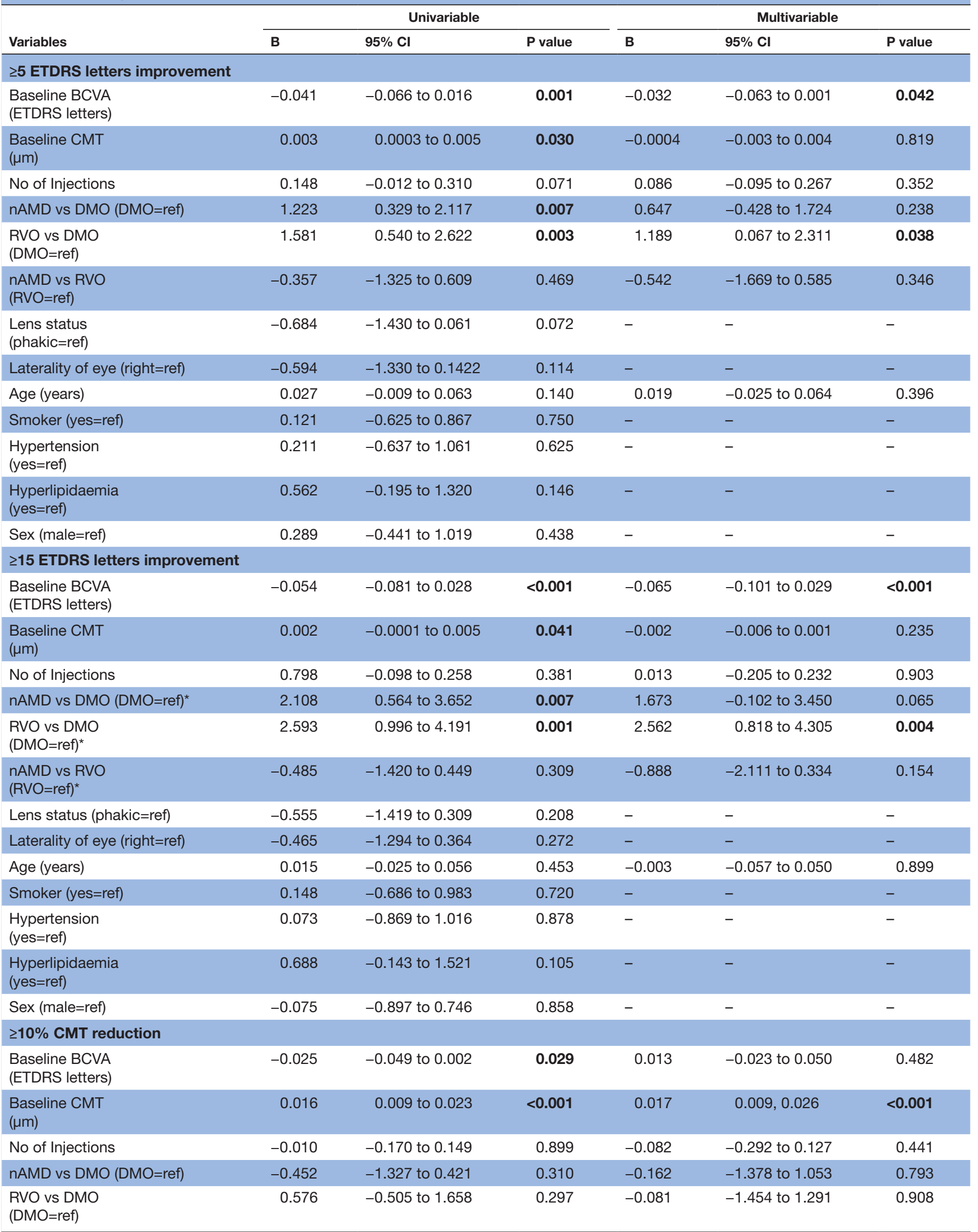




\section{Table 4 Continued}

\begin{tabular}{|c|c|c|c|c|c|c|}
\hline \multirow[b]{2}{*}{ Variables } & \multicolumn{3}{|c|}{ Univariable } & \multicolumn{3}{|c|}{ Multivariable } \\
\hline & B & $95 \% \mathrm{Cl}$ & $P$ value & B & $95 \% \mathrm{Cl}$ & $P$ value \\
\hline $\begin{array}{l}\text { nAMD vs RVO } \\
\text { (RVO=ref) }\end{array}$ & -1.02 & -2.041 to 0.016 & 0.046 & -0.081 & -1.433 to 1.271 & 0.906 \\
\hline $\begin{array}{l}\text { Lens status } \\
\text { (phakic=ref) }\end{array}$ & 0.001 & -0.760 to 0.763 & 0.997 & - & - & - \\
\hline Laterality of eye (right=ref) & 0.100 & -0.651 to 0.852 & 0.793 & - & - & - \\
\hline Age (years) & -0.029 & -0.067 to 0.008 & 0.132 & -0.008 & -0.060 to 0.044 & 0.766 \\
\hline Smoker (yes=ref) & 0.296 & -0.481 to 1.075 & 0.455 & - & - & - \\
\hline $\begin{array}{l}\text { Hypertension } \\
\text { (yes=ref) }\end{array}$ & -0.641 & -1.494 to 0.212 & 0.141 & - & - & - \\
\hline $\begin{array}{l}\text { Hyperlipidaemia } \\
\text { (yes=ref) }\end{array}$ & -0.473 & -1.239 to 0.292 & 0.226 & - & - & - \\
\hline Sex (male=ref) & -0.328 & -1.086 to 0.429 & 0.396 & - & - & - \\
\hline
\end{tabular}

Significant $p$ values are bolded.

*Firth-logistics; multivariable=adjusted for baseline BCVA, baseline CMT, injection number, age and disease type.

BCVA, best-corrected visual acuity; CMT, central macular thickness; DMO, diabetic macular oedema; ETDRS, early treatment diabetic retinopathy study; nAMD, neovascular age-related macular degeneration; RVO, retinal vein occlusion.

groups. Previous studies have found favourable outcomes in younger age groups, though the exact mechanisms for this are unknown. It may be partly due to the fact that the macula in young patients is better able to tolerate structural and functional damage. ${ }^{21}{ }^{30}$ On the contrary, the improvement in vision in our DMO group was significantly lower despite being a younger cohort. Also, DMO patients in our study received significantly fewer injections (median=9) compared with nAMD (median=10) or RVO (median=10) patients, which could be one of the reasons for a suboptimal outcome in the DMO group. While statistically significant, the difference of one injection over 12 months is likely not clinically meaningful. Interestingly, the frequency of injection in our study was similar to a number of RCTs for DMO (median $=8-9)^{31} 32$ and higher than other real-world studies (median=5$8),{ }^{33-35}$ thus ruling out undertreatment. In addition, DMO is a highly complex disease and there may be other unexplored causes of poor outcomes in DMO patients, including genetic, epigenetic and environmental factors. $^{36}$

This study is consistent with a similar study by Wecker $e t$ $a l .{ }^{37}$ They reported a higher proportion of RVO patients (24\%) gained more than 15 ETDRS letters than DMO $(13.9 \%)$ or nAMD $(14.1 \%)$ patients after 12 months. While the proportion of nAMD and RVO patients with 15 letters improvement was higher in our study (RVO:43\%, nAMD:32\%), fewer of our DMO patients (5\%) reached this goal. A Thai study by Kumluang et $a l^{88}$ evaluated the effectiveness of anti-VEGF injections in various retinal conditions, including nAMD, DMO and RVO. Again, RVO patients $(63 \%)$ represented the group with the highest visual acuity gain ( $>10$ ETDRS letters improvement) compared with other groups (nAMD $=46 \%, \mathrm{DMO}=46 \%)$. Patients in that study were only followed up for 6 months. Not all patients benefit immediately and it has been suggested that patients should be treated for at least 12 months before assessing the treatment response. ${ }^{39}$ In a study by Ehlken $e t a l,{ }^{40}$ the authors compared compliance between nAMD, DMO and RVO. DMO patients had the highest risk of non-adherence, leading to worse vision outcomes. Poor patient compliance could be a reason for smaller gains in our DMO patients although we could not evaluate compliance owing to a retrospective design. Patients with diabetes may be focused on other life-threatening diabetic complications requiring more urgent treatment, thus promoting poor compliance with the monthly anti-VEGF regimens.

Change in CMT was not included in either of the previous studies. BCVA is the preferred measure of response, but it can be highly subjective and influenced by a range of confounding factors including the refractive status of the eye or presence of cataract. CMT can be objectively measured and is a direct measure of the effect of the drug on the target tissue. As there is limited correlation between the two measures, ${ }^{41}$ it is useful to use both to define response. In our study, similar conclusions were drawn from both measures of treatment response.

Limitations of this study include small and unequal sample sizes between the three disease, the retrospective nature and exclusion of patients due to missing data. Baseline characteristics between the three eye diseases were not equal, though they were included as covariates in the analyses. Further, no single type of anti-VEGF drug was used consistently in these cohorts who were treated at the clinician's discretion. Many patients received two or three different anti-VEGF agents over the course of 12 months, reflecting real-world practices. Despite a small subcohort size, analysing outcomes based on the type of anti-VEGF drug (online supplemental file S1) also showed greater vision improvement in RVO and nAMD compared with DMO patients. A more detailed analysis stratified by injection type was not possible owing to the small sample size. 
When comparing anatomical response, we should be mindful that CMT is not the only measurable anatomical parameter. The anatomical responses in the three disorders are quite varied and evaluation of other parameters (such as presence of active macular bleeding, size of neovascular membrane, angiographic features, and pattern of macular oedema) could provide greater insights into disease outcomes. ${ }^{42}{ }^{43}$ Further, though OCT measurement is considered fundamental in both clinical care and research, several patient-related (media opacity, patient cooperation, macular contour changes) and software-related (machine resolution, autosegmentation algorithm) factors can affect the repeatability of OCT measurements, which may cause serious errors in the interpretation of OCT parameters. ${ }^{44} 45$ A more comprehensive analysis of these factors was not possible due to lack of relevant data in the retrospective study design. Finally, although nAMD, DMO and RVO share VEGF as a major driving force, each of these diseases has distinct pathophysiology and subtypes. Consequently, the appropriateness of statistically combining and comparing the results warrants precaution.

In conclusion, this study aimed to compare visual outcomes between three common retinal conditions treated with anti-VEGF injections. We demonstrate that nAMD and RVO patients had greater improvements in visual gain, but DMO patients had better vision at baseline and after 12 months. While there was little room for improvement in DMO patients, the anti-VEGF drugs likely prevent further vision loss. nAMD and RVO patients had lower baseline vision but were able to reach comparable vision after 12 months of treatment. Diagnosing and treating these groups at an earlier time point might improve their final vision further. Notably, a larger, balanced study design including patients with comparable baseline vision and CMT across all three disease groups would help to explore the possible role of a ceiling/floor effect further. Finally, it would be interesting to explore the long-term difference in outcomes of anti-VEGF treatment in nAMD, DMO and RVO patients given the chronic nature of the diseases. An extended study with a longer follow-up would address this.

\section{Author affiliations}

${ }^{1}$ Menzies Institute for Medical Research, University of Tasmania, Hobart, TAS, Australia

${ }^{2}$ Department of Ophthalmology, University of Tasmania, Hobart, Tasmania, Australia

Acknowledgements We would like to acknowledge all the clinical staffs and patients of Royal Hobart Hospital (ophthalmology department) and Hobart Eye Surgeons for their support during patient recruitment.

Contributors RLG, KPB, LMF, BJM and NV conceived the concept for this study. RLG performed the statistical analysis and wrote the initial draft of the manuscript. LMF, BJM, AWH, NV and KPB were involved in critical revision of the manuscript.

Funding The study was funded by the Tasmanian Community Fund and the National Health and Medical Research Council (NHMRC) Australia Centre for Research Excellence (GNT1116360). RLG is supported by a scholarship from Diabetes Tasmania and the Patricia F Gordon Postgraduate Top-Up Scholarship in Medical Research. LMF is supported by a Cancer Council Tasmania/College of Health and Medicine, University of Tasmania Fellowship. KPB is supported by NHMRC Senior Research Fellowship.

Competing interests None declared.

\section{Patient consent for publication Not required.}

Ethics approval The study adhered to the tenets of the Declaration of Helsinki and was approved through the Human Research Ethics Committee of the University of Tasmania (approval number H0012902).

Provenance and peer review Not commissioned; externally peer reviewed.

Data availability statement Data are available upon request.

Supplemental material This content has been supplied by the author(s). It has not been vetted by BMJ Publishing Group Limited (BMJ) and may not have been peer-reviewed. Any opinions or recommendations discussed are solely those of the author(s) and are not endorsed by BMJ. BMJ disclaims all liability and responsibility arising from any reliance placed on the content. Where the content includes any translated material, BMJ does not warrant the accuracy and reliability of the translations (including but not limited to local regulations, clinical guidelines, terminology, drug names and drug dosages), and is not responsible for any error and/or omissions arising from translation and adaptation or otherwise.

Open access This is an open access article distributed in accordance with the Creative Commons Attribution Non Commercial (CC BY-NC 4.0) license, which permits others to distribute, remix, adapt, build upon this work non-commercially, and license their derivative works on different terms, provided the original work is properly cited, appropriate credit is given, any changes made indicated, and the use is non-commercial. See: http://creativecommons.org/licenses/by-nc/4.0/.

\section{ORCID iDs}

Rajya L Gurung http://orcid.org/0000-0003-0816-9823

Bennet J McComish http://orcid.org/0000-0003-1940-0007

\section{REFERENCES}

1 Drolet DW, Nelson J, Tucker CE, et al. Pharmacokinetics and safety of an anti-vascular endothelial growth factor aptamer (NX1838) following injection into the vitreous humor of rhesus monkeys. Pharm Res 2000;17:1503-10.

2 Rajappa M, Saxena P, Kaur J. Ocular angiogenesis: mechanisms and recent advances in therapy. Adv Clin Chem 2010;50:103-21.

3 Wong WL, Su X, Li X, et al. Global prevalence of age-related macular degeneration and disease burden projection for 2020 and 2040: a systematic review and meta-analysis. Lancet Glob Health 2014;2:e106-16.

4 Ambati J, Fowler BJ. Mechanisms of age-related macular degeneration. Neuron 2012;75:26-39.

5 Kvanta A, Algvere PV, Berglin L, et al. Subfoveal fibrovascular membranes in age-related macular degeneration express vascular endothelial growth factor. Invest Ophthalmol Vis Sci 1996;37:763-34.

6 Wenick AS, Bressler NM. Diabetic macular edema: current and emerging therapies. Middle East Afr J Ophthalmol 2012;19:4.

7 Romero-Aroca P, Baget-Bernaldiz M, Pareja-Rios A, et al. Diabetic macular edema pathophysiology: vasogenic versus inflammatory. $J$ Diabetes Res 2016;2016:1-17.

8 Yenihayat F, Özkan B, Kasap M, et al. Vitreous IL-8 and VEGF levels in diabetic macular edema with or without subretinal fluid. Int Ophthalmol 2019;39:821-8.

9 Funatsu $\mathrm{H}$, Yamashita $\mathrm{H}$, Noma $\mathrm{H}$, et al. Increased levels of vascular endothelial growth factor and interleukin-6 in the aqueous humor of diabetics with macular edema. Am J Ophthalmol 2002;133:70-7.

10 Boulton M, Foreman D, Williams G, et al. Vegf localisation in diabetic retinopathy. Br J Ophthalmol 1998;82:561-8.

11 Laouri M, Chen E, Looman M, et al. The burden of disease of retinal vein occlusion: review of the literature. Eye 2011;25:981-8.

12 Noma H, Funatsu $\mathrm{H}$, Yamasaki M, et al. Pathogenesis of macular edema with branch retinal vein occlusion and intraocular levels of vascular endothelial growth factor and interleukin-6. Am J Ophthalmol 2005;140:256.e1-256.e7.

13 Gregori NZ, Feuer W, Rosenfeld PJ. Novel method for analyzing Snellen visual acuity measurements. Retina 2010;30:1046-50.

14 Diabetic Retinopathy Clinical Research Network, Wells JA, Glassman AR, et al. Aflibercept, bevacizumab, or ranibizumab for diabetic macular edema. N Engl J Med 2015;372:1193-203.

15 Ishibashi T, Li X, Koh A, et al. The reveal study: ranibizumab monotherapy or combined with laser versus laser monotherapy in Asian patients with diabetic macular edema. Ophthalmology 2015;122:1402-15 
16 Ohji M, Takahashi K, Okada AA, et al. Efficacy and safety of intravitreal aflibercept treat-and-extend regimens in exudative age-related macular degeneration: 52- and 96-week findings from ALTAIR : a randomized controlled trial. Adv Ther 2020;37:1173-87.

17 Brown DM, Campochiaro PA, Bhisitkul RB, et al. Sustained benefits from ranibizumab for macular edema following branch retinal vein occlusion: 12-month outcomes of a phase III study. Ophthalmology 2011;118:1594-602.

18 Ciulla TA, Bracha P, Pollack J, et al. Real-world outcomes of antivascular endothelial growth factor therapy in diabetic macular edema in the United States. Ophthalmol Retina 2018;2:1179-87.

19 Shimura M, Kitano S, Muramatsu D, et al. Real-world management of treatment-naïve diabetic macular oedema in Japan: two-year visual outcomes with and without anti-VEGF therapy in the STREATDME study. Br J Ophthalmol 2020:104:1209-15.

20 Mitchell P, Chong V. Baseline predictors of 3-year responses to ranibizumab and laser photocoagulation therapy in patients with visual impairment due to diabetic macular edema (DME): the restore study. Invest Ophthalmol Vis Sci 2013;54:2373-73.

21 Kaiser PK, Brown DM, Zhang K, et al. Ranibizumab for predominantly classic neovascular age-related macular degeneration: subgroup analysis of first-year anchor results. Am J Ophthalmol 2007:144:e4:850-7.

22 Özkaya A, Karabaș L, Alagöz C, et al. Real-world outcomes of antiVEGF treatment for neovascular age-related macular degeneration in turkey: a multicenter retrospective study, Bosphorus retina Study Group report no: 1. Turk J Ophthalmol 2018;48:232-7.

23 Vaz-Pereira S, Marques IP, Matias J, et al. Real-world outcomes of anti-VEGF treatment for retinal vein occlusion in Portugal. Eur $J$ Ophthalmol 2017;27:756-61.

24 Korobelnik J-F, Daien V, Faure C, et al. Real-world outcomes following 12 months of intravitreal aflibercept monotherapy in patients with diabetic macular edema in France: results from the APOLLON study. Graefes Arch Clin Exp Ophthalmol 2020;258:521-8.

25 Dugel PU, Hillenkamp J, Sivaprasad S, et al. Baseline visual acuity strongly predicts visual acuity gain in patients with diabetic macular edema following anti-vascular endothelial growth factor treatment across trials. Clin Ophthalmol 2016;10:1103.

26 Sophie R, Lu N, Campochiaro PA. Predictors of functional and anatomic outcomes in patients with diabetic macular edema treated with ranibizumab. Ophthalmology 2015;122:1395-401.

27 Amoaku WM, Chakravarthy U, Gale R, et al. Defining response to anti-VEGF therapies in neovascular AMD. Eye 2015;29:721-31.

28 Mitchell P, Bandello F, Schmidt-Erfurth U, et al. The restore study: ranibizumab monotherapy or combined with laser versus laser monotherapy for diabetic macular edema. Ophthalmology 2011;118:615-25.

29 Ying G-S, Maguire MG, Pan W, et al. Baseline predictors for five-year visual acuity outcomes in the comparison of AMD treatment trials. Ophthalmol Retina 2018;2:525-30.

30 Bressler SB, Qin H, Beck RW, et al. Factors associated with changes in visual acuity and central subfield thickness at 1 year after treatment for diabetic macular edema with ranibizumab. Arch Ophthalmol 2012;130:1153-61.
31 Michaelides M, Kaines A, Hamilton RD, et al. A prospective randomized trial of intravitreal bevacizumab or laser therapy in the management of diabetic macular edema (bolt study) 12-month data: report 2. Ophthalmology 2010;117:e2:1078-86.

32 Diabetic Retinopathy Clinical Research Network, Elman MJ, Aiello LP, et al. Randomized trial evaluating ranibizumab plus prompt or deferred laser or triamcinolone plus prompt laser for diabetic macular edema. Ophthalmology 2010;117:e35:1064-77.

33 Bhandari S, Nguyen V, Fraser-Bell S, et al. Ranibizumab or aflibercept for diabetic macular edema: comparison of 1-year outcomes from the fight retinal blindness! registry. Ophthalmology 2020;127:608-15.

34 Ciulla TA, Pollack JS, Williams DF. Visual acuity outcomes and antiVEGF therapy intensity in diabetic macular oedema: a real-world analysis of 28658 patient eyes. Br J Ophthalmol 2021;105:216-21.

35 Urbančič M, Klobučar P, Zupan M, et al. Anti-Vegf treatment of diabetic macular edema: two-year visual outcomes in routine clinical practice. J Ophthalmol 2020;2020:1-8.

36 Gurung RL, FitzGerald LM, McComish BJ, et al. Identifying genetic risk factors for diabetic macular edema and the response to treatment. J Diabetes Res 2020;2020:1-12.

37 Wecker T, Ehlken C, Bühler A, et al. Five-year visual acuity outcomes and injection patterns in patients with pro-re-nata treatments for AMD, DME, RVO and myopic CNV. Br J Ophthalmol 2017;101:353-9.

38 Kumluang S, Ingsrisawang L, Sangroongruangsri S, et al. A real-world study of effectiveness of intravitreal bevacizumab and ranibizumab injection for treating retinal diseases in Thailand. BMC Ophthalmol 2019;19:82.

39 Chatziralli I, Santarelli M, Patrao N, et al. Identification of time point to best define 'sub-optimal response' following intravitreal ranibizumab therapy for diabetic macular edema based on real-life data. Eye 2017;31:1594-9.

40 Ehlken C, Helms M, Böhringer D, et al. Association of treatment adherence with real-life Va outcomes in AMD, DME, and BRVO patients. Clin Ophthalmol 2018;12:13-20.

41 Diabetic Retinopathy Clinical Research Network, Browning DJ, Glassman AR, et al. Relationship between optical coherence tomography-measured central retinal thickness and visual acuity in diabetic macular edema. Ophthalmology 2007;114:525-36.

42 Ashraf M, Souka A, Adelman RA. Age-related macular degeneration: using morphological predictors to modify current treatment protocols. Acta Ophthalmol 2018;96:120-33.

43 Wu P-C, Lai C-H, Chen C-L, et al. Optical coherence tomographic patterns in diabetic macula edema can predict the effects of intravitreal bevacizumab injection as primary treatment. J Ocul Pharmacol Ther 2012;28:59-64.

44 Lee H-J, Kim M-S, Jo Y-J, et al. Ganglion cell-inner plexiform layer thickness in retinal diseases: repeatability study of spectraldomain optical coherence tomography. Am J Ophthalmol 2015;160:e1:283-9.

45 Hong $\mathrm{EH}$, Ryu SJ, Kang MH, et al. Comparison of repeatability of swept-source and spectral-domain optical coherence tomography for measuring inner retinal thickness in retinal disease. PLoS One 2019;14:e0210729. 